\title{
Recent progress on nuclear parton distribution functions
}

\author{
M. Hirai*, S. Kumano ${ }^{\dagger}$ and K. Saito* \\ ${ }^{*}$ Department of Physics, Faculty of Science and Technology, Tokyo University of Science \\ 2641, Yamazaki, Noda, Chiba, 278-8510, Japan \\ ${ }^{\dagger}$ KEK Theory Center, Institute of Particle and Nuclear Studies, KEK \\ and Department of Particle and Nuclear Studies, Graduate University for Advanced Studies \\ 1-1, Ooho, Tsukuba, Ibaraki, 305-0801, Japan
}

\begin{abstract}
We report current status of global analyses on nuclear parton distribution functions (NPDFs). The optimum NPDFs are determined by analyzing high-energy nuclear reaction data. Due to limited experimental measurements, antiquark modifications have large uncertainties at $x>0.2$ and gluon modifications cannot be determined. A nuclear modification difference between $u$ and $d$ quark distributions could be an origin of the long-standing NuTeV $\sin ^{2} \theta_{w}$ anomaly. There is also an issue of nuclear modification differences between the structure functions of charged-lepton and neutrino reactions. Next, nuclear clustering effects are discussed in structure functions $F_{2}^{A}$ as a possible explanation for an anomalous result in the ${ }^{9} \mathrm{Be}$ nucleus at the Thomas Jefferson National Accelerator Facility (JLab). Last, tensor-polarized quark and antiquark distribution functions are extracted from HERMES data on the polarized structure function $b_{1}$ of the deuteron, and they could be used for testing theoretical models and for proposing future experiments, for example, the one at JLab. Such measurements could open a new field of spin physics in spin-one hadrons.
\end{abstract}

Keywords: Quark, gluon, parton, distribution, QCD, nuclear effect

PACS: 13.60.Hb, 13.60.-r, 24.85.+p, 25.30.-c, 13.88.+e

\section{INTRODUCTION}

Parton distribution functions (PDFs) of the nucleon have been investigated for a long time, and they are now established except for extremely small- $x$ and large- $x$ regions. A precise determination of the PDFs is very important for new discoveries at LHC (Large Hadron Collider). For heavy-ion collisions at LHC, nuclear parton distribution functions (NPDFs) should be also determined precisely because 10-20\% nuclear modifications exist in medium-size nuclei and they are larger in heavy nuclei. In addition to heavy-ion physics at LHC, an accurate determination of the NPDFs is valuable for other topics such as establishment of non-perturbative aspect of QCD by comparisons with theoretical models of nuclear medium effects, neutrino-oscillation studies by nuclear corrections in ${ }^{16} \mathrm{O}$, high-energy cosmic-ray interactions with air for studying GZK (Greisen-ZatsepinKuzmin) cutoff, and determination of fundamental constants like $\sin ^{2} \theta_{W}$. In contrast to the nucleonic PDFs, the NPDFs have not been determined well, which adds uncertainties in investigating the above-mentioned topics. In this report, we explain the current status of the NPDF determination and our recent studies on nuclear structure functions.

Nuclear modifications of the structure function $F_{2}$ have been investigated in deep inelastic lepton scattering from nuclear targets [1]. Now, we have measurements from relatively small $x$ to large $x$ with a variety of nuclei from the deuteron to heavy ones. Us- 
ing these data together with Drell-Yan measurements of nuclear targets, we determined the NPDFs. RHIC (Relativistic Heavy Ion Collider) and neutrino reaction data could be used in addition. First, we introduce the current status of the NPDF determination $[2,3,5,4,6,7]$ and a possible relation to the NuTeV $\sin ^{2} \theta_{W}$ anomaly $[8,9,10]$. We also discuss an issue of different nuclear modifications between charged-lepton and neutrino reactions. Second, the structure function $F_{2}$ of ${ }^{9} \mathrm{Be}$ is discussed for explaining an anomalous JLab data on the nuclear modification slope $d\left(F_{2}^{B e} / F_{2}^{D}\right) / d x[11]$. Since ${ }^{9} \mathrm{Be}$ is a nucleus which is usually described by clustering structure of two $\alpha$ nuclei with neutron clouds, the data could indicate a clustering feature in the deep inelastic scattering (DIS) [12]. Third, new spin structure of the deuteron is investigated by analyzing HERMES data on the tensor-structure function $b_{1}[13,14]$. The tensor structure of nuclei and hadrons in the high-energy region is an unexplored topic, although it has been investigated extensively at low energies in connection with nuclear tensor force due to meson exchanges. It is interesting to understand the tensor structure in terms of quark and gluon degrees of freedom. Since $b_{1}$ vanishes if constituents are in the $S$-wave, it could also be a good probe of quark-gluon dynamics including orbital angular momenta.

\section{NUCLEAR PARTON DISTRIBUTION FUNCTIONS}

Nuclear modifications of the structure function $F_{2}$ are typically $10-20 \%$ for medium size nuclei. Therefore, it is convenient to express the NPDFs by the corresponding nucleonic PDFs multiplied by nuclear modification factors $w_{i}\left(i=u_{v}, d_{v}, \bar{q}, g\right)$ :

$$
f_{i}^{A}\left(x, Q_{0}^{2}\right)=w_{i}(x, A, Z) \frac{1}{A}\left[Z f_{i}^{p}\left(x, Q_{0}^{2}\right)+N f_{i}^{n}\left(x, Q_{0}^{2}\right)\right]
$$

where $p$ and $n$ indicate the proton and neutron, $A, Z$, and $N$ are mass, atomic, and neutron numbers, respectively, and $Q_{0}^{2}$ is the initial $Q^{2}$ scale. The isospin symmetry $\left(d^{n}=u^{p}, u^{n}=d^{p}\right)$ is assumed in relating the distributions of the neutron to the ones of the proton. It should be noted that the above parametrization cannot describe the NPDFs at $x>1$ because the distributions vanish in the nucleon, whereas the kinematical range is $0<x<A$ in a nucleus.

There are a few groups which have been investigating the optimum NPDFs by global analyses of world data on high-energy nuclear reactions $[2,3,5,4,6,7]$. The $x$ dependent functional forms are different depending on the analysis groups. For example, the HKNS parametrization uses [2]

$$
w_{i}(x, A, Z)=1+\left(1-\frac{1}{A^{\alpha}}\right) \frac{a_{i}+b_{i} x+c_{i} x^{2}+d_{i} x^{3}}{(1-x)^{\beta}},
$$

at $Q_{0}^{2}=1 \mathrm{GeV}^{2}$. Here, $\alpha, \beta, a_{i}, b_{i}, c_{i}$, and $d_{i}$ are parameters to be determined by a $\chi^{2}$ analysis. Other groups' functional forms are listed in Ref. [15]. In the parametrization of de Florian and Sassot [3], the NPDFs are expressed by convolution of the nucleonic PDFs with a modification function $w(x)$ and a small initial $Q^{2}$ scale $\left(0.4 \mathrm{GeV}^{2}\right)$ is used for utilizing the GRV-type PDFs. In the analysis of Eskola, Paukkunen, and Salgado (EPS) [4], the $x$ region is divided into three: shadowing, anti-shadowing, and EMC \& 
Fermi-motion regions, and different parameters are assigned to the three functions in each NPDF. Schienbein et al.'s analysis used a functional form of polynomials of $x$ combined with exponential functions [5]. Used data sets are similar; however, the EPS [4] included some of the RHIC data and Schienbein et al. [5] also used NuTeV neutrino data. There are other works by Kulagin and Petti (KP) [6] and Frankfurt, Guzey, and Strikman (FGS) [7]. The KP method is to determine off-shell structure functions of the nucleon by calculating other nuclear modifications in conventional models with binding, Fermi motion, and multiple scattering. The FGS method is mainly intended to describe the small- $x$ shadowing part by the multiple scattering.

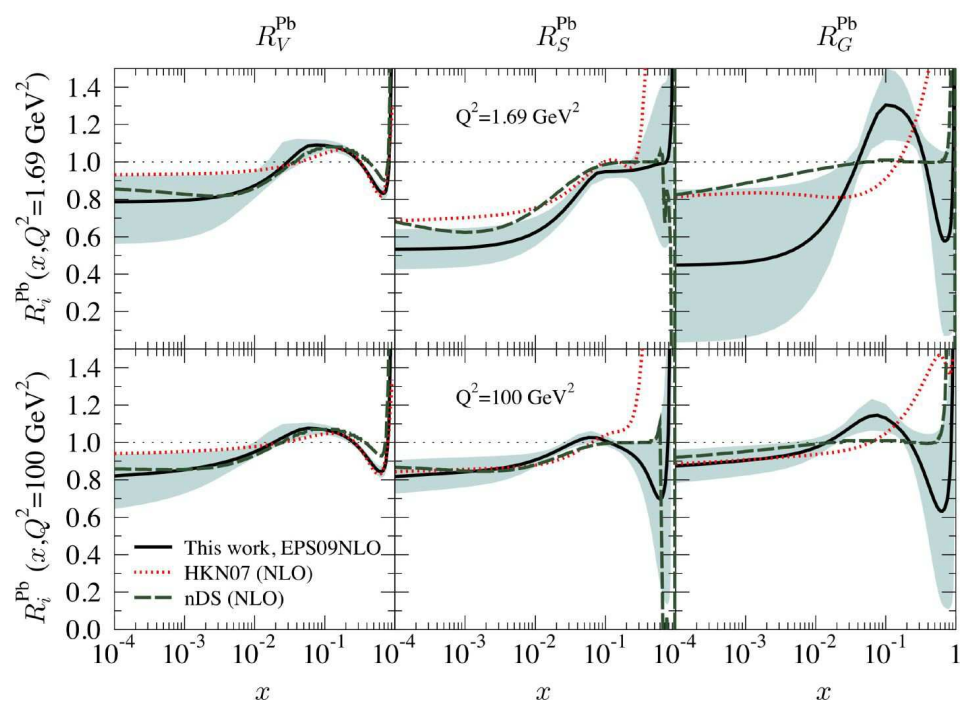

FIGURE 1. Nuclear modifications of the PDFs are shown for the lead nucleus at $Q^{2}=1.69$ and $100 \mathrm{GeV}^{2}$ [4]. The functions $R_{V}^{P b}, R_{S}^{P b}$, and $R_{G}^{P b}$ indicate the valence-quark, sea-quark, and gluon modifications, respectively. Three parametrization results (EPS09, HKN07, and nDS) are shown with uncertainty bands.

Nuclear modifications of the PDFs are determined by $\chi^{2}$ analyses of world data on the structure-function $F_{2}$ ratios $F_{2}^{A} / F_{2}^{A^{\prime}}$, Drell-Yan cross-section ratios $\sigma_{D Y}^{p A} / \sigma_{D Y}^{p A^{\prime}}$, RHIC $d A$ data, and neutrino-DIS structure functions. Typical results are shown in Fig. 1 for nuclear modifications of the lead nucleus at $Q^{2}=1.69$ and $100 \mathrm{GeV}^{2}$. Three different parametrizations are shown together with uncertainty bands. Although the three groups used different parametrizations for the $x$ dependence and slightly different data sets, the determined distributions roughly agree with each other within the uncertainty bands. There are large uncertainties in the antiquark distributions at medium and large $x$ and in the gluon distribution at whole $x$. They should be determined by future experimental measurements.

The studies of nuclear modifications could affect the NuTeV anomaly on the weakmixing angle. The NuTeV anomaly has been a long-standing issue that the mixing angle measured by the NuTeV neutrino DIS, $\sin ^{2} \theta_{W}=0.2277 \pm 0.0013$ (stat) \pm 0.0009 (syst), is different from other measurements, $\sin ^{2} \theta_{W}=0.2227 \pm 0.0004$ [8]. In extracting the mixing angle from neutrino DIS, there is a Paschos-Wolfenstein (PW) relation for the isoscalar nucleon. There are various corrections to use it for the iron target in the $\mathrm{NuTeV}$ 
experiment:

$$
\frac{\sigma_{N C}^{v A}-\sigma_{N C}^{\bar{v} A}}{\sigma_{C C}^{v A}-\sigma_{C C}^{\bar{v} A}}=\frac{1}{2}-\sin ^{2} \theta_{W}+(Z \neq N)+\left(w_{u_{v}} \neq w_{d_{v}}\right)+\left(u^{p} \neq d^{n}\right)+(s \neq \bar{s})+(c \neq \bar{c}),
$$

where $\sigma_{C C}^{V A}$ and $\sigma_{N C}^{V A}$ are charged-current $(\mathrm{CC})$ and neutral-current (NC) cross sections, respectively, in neutrino-nucleus DIS. The parentheses $(\cdots)$ indicate various corrections to the PW relation. The obvious non-isoscalar correction $(Z \neq N)$ is taken into account in the NuTeV analysis, so that it should not be the origin of the anomaly.

First, since the NuTeV target is the iron nucleus, the anomaly could come from nuclear modification difference between $u_{v}$ and $d_{v}$. In particular, baryon-number and charge conservations indicate that the modifications should be different between $u_{v}$ and $d_{v}$ [9]. However, the current data are not enough to probe such a small effect by a global NPDF analysis [9]. According to Cloët, Bentz, and Thomas, the nuclear modification is larger for $u$ quark than the one for $d$ due to vector mean field which is adjusted to explain the symmetry energy of a nucleus [10]. Future pion-induced DrellYan measurements such as by the COMPASS collaboration could be used for finding the difference [16]. Second, QED effects on the PDFs, namely $u^{p} \neq d^{n}$ and $d^{p} \neq u^{n}$ could be the origin of the anomaly. The symmetry relations between the proton and neutron PDFs $\left(u^{p}=d^{n}, d^{p}=u^{n}\right)$ are usually assumed in all the global analyses by considering that the QED effects are less than a few percent $\left(\sim \alpha / \alpha_{s}\right)$. A possible QCD effect was investigated in Ref. [17] by somewhat assuming a photon distribution at the initial scale $Q^{2}=1 \mathrm{GeV}^{2}$. The result indicated that the anomaly could come from such an effect although a precise determination of the QED effect is not possible at this stage. Third, the strange and charm asymmetries $(s \neq \bar{s}, c \neq \bar{c})$ could be origins. However, such effects cannot be determined from experimental measurements at this stage in the sense that the distribution $s(x)-\bar{s}(x)$ still has a large uncertainty band [18].

Another issue of the current NPDFs is the modification differences between charged-lepton and neutrino reactions [5]. The nuclear modifications of $F_{2}$ are well established in charged-lepton reactions, and the optimum NPDFs are extracted mainly from their data. However, the modifications are very different in neutrino DIS as shown by the solid curve in Fig. 2 [5], whereas the other curves are the modifications suggested by chargedlepton DIS. The medium- $x(=0.5 \sim 0.8)$ modifications are different and the antishadowing phenomenon does not appear at $x=0.1 \sim 0.2$ in the neutrino DIS. Since

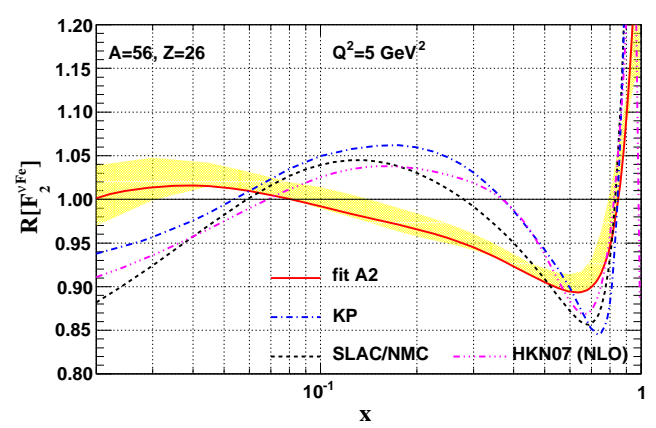

FIGURE 2. The solid curve (fit A2) indicates the nuclear modification of $F_{2}$ (iron) in neutrino DIS [5]. For comparison, two parametrization results (KP and HKN07) are shown together with the modification in charged-lepton DIS (SLAN/NMC).

neutrino data are partially used for extracting current "nucleonic" PDFs, this issue should be solved for accurate determinations of not only the NPDFs but also the PDFs of the nucleon. 


\section{CLUSTERING EFFECTS IN NUCLEAR STRUCTURE FUNCTIONS}

It is known in nuclear-structure studies that some light nuclei exhibit phenomena of nuclear clustering. For example, the ${ }^{8}$ Be nucleus is theoretically described by two $\alpha$ like clusters according to a Monte Carlo calculation [19] rather than the usual monotonic density distribution estimated by a shell model. This fact led us to wonder whether such clustering structure could appear in high-energy reactions, for example, in the structure functions $F_{2}^{A}$. Although simple multiquark clusters were investigated at the early stage of EMC-effect studies, clustering aspects of nucleons were not investigated. Recently, such a signature could be found by a JLab experiment [11] according to the theoretical work of Ref. [12]. We explain this theoretical approach on the clustering effects.

First, we estimate effects of nuclear clustering by using a conventional convolution approach. A nuclear structure function $F_{2}^{A}$ is given by the nucleonic one convoluted with a nucleon momentum distribution in the nucleus $f(y):[1,20]$

$F_{2}^{A}\left(x, Q^{2}\right)=\int_{x}^{A} d y f(y) F_{2}^{N}\left(x / y, Q^{2}\right), f(y)=\frac{1}{A} \int d^{3} p_{N} y \delta\left(y-\frac{p_{N} \cdot q}{M_{N} v}\right)\left|\phi\left(\vec{p}_{N}\right)\right|^{2}$,

where $F_{2}^{N}$ is the structure function for the nucleon, and $y$ is the momentum fraction $y=$ $M_{A} p_{N} \cdot q /\left(M_{N} p_{A} \cdot q\right) \simeq A p_{N}^{+} /\left(p_{A}^{+}\right)$with a light-cone momentum $p^{+}$. If the momentum density $\left|\phi\left(\vec{p}_{N}\right)\right|^{2}$ is obtained, $F_{2}^{A}$ can be calculated by this equation.

Two theoretical models are used for calculating the nuclear densities. In order to investigate a possible clustering effect, an antisymmetrized molecular dynamics (AMD) is used and a simple shell model is also employed to compare with AMD calculations. The AMD is a variational approach for describing nuclei. Its advantage is that there is no a priori assumption on nuclear structure whether it is a shell or cluster-like configuration. A nuclear wave function is given by the Slater determinant of single-particle wave packets: $\left|\Phi\left(\vec{r}_{1}, \vec{r}_{2}, \cdots, \vec{r}_{A}\right)\right\rangle=(1 / \sqrt{A !}) \operatorname{det}\left[\varphi_{1}\left(\vec{r}_{1}\right), \varphi_{2}\left(\vec{r}_{2}\right), \cdots, \varphi_{A}\left(\vec{r}_{A}\right)\right]$ where $\varphi_{i}\left(\vec{r}_{j}\right)$ is the single-particle wave function given by $\varphi_{i}\left(\vec{r}_{j}\right)=\phi_{i}\left(\vec{r}_{j}\right) \chi_{i} \tau_{i}$ with spin and isospin states $\chi_{i}$ and $\tau_{i}$. The function $\phi_{i}\left(\vec{r}_{j}\right)$ is the space part expressed as $\phi_{i}\left(\vec{r}_{j}\right)=(2 v / \pi)^{3 / 4} \exp \left[-v\left(\vec{r}_{j}-\right.\right.$ $\left.\left.\vec{Z}_{i} / \sqrt{v}\right)^{2}\right]$, where $v$ and $\vec{Z}_{i}$ are variational parameters. Simple effective $N N$ interactions are used for describing gross properties of nuclei. The variational parameters are then

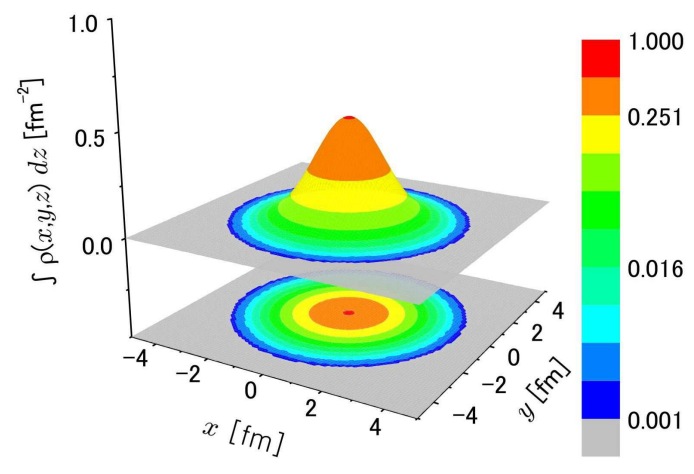

FIGURE 3. Two-dimensional $(x, y)$ coordinatespace density of ${ }^{4} \mathrm{He}$ by the AMD [12]. The density is shown by taking an integral over the coordinate $z: \int d z \rho(x, y, z)$. The shell-model density is almost the same as the above distribution in ${ }^{4} \mathrm{He}$.

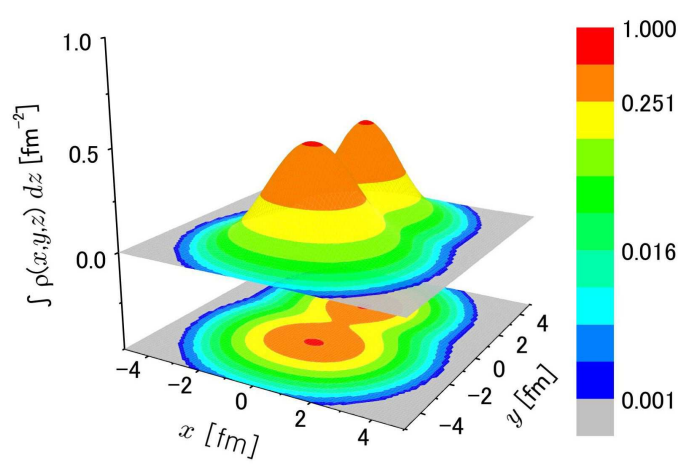

FIGURE 4. Two-dimensional $(x, y)$ coordinatespace density of ${ }^{9} \mathrm{Be}$ by the AMD [12]. The notations are the same as the one in Fig. 3. The shellmodel density is a monotonic distribution, which is different from the above distribution. 
determined by minimizing the system energy with a frictional-cooling method. Obtained space densities are shown in Figs. 3 and 4 for ${ }^{4} \mathrm{He}$ and ${ }^{9} \mathrm{Be}$. Figure 3 indicates that the ${ }^{4} \mathrm{He}$ density is a monotonic distribution as typically suggested by shell models. However, it is obvious from Fig. 4 that the ${ }^{9} \mathrm{Be}$ distribution is totally different. There are two density peaks which roughly correspond to two $\alpha$ clusters with surrounding neutron clouds. It is interesting to investigate that such a nuclear clustering could be found in high-energy experiments, for example, in nuclear structure functions because the anomalous EMC effect was indicated by the JLab experiment for ${ }^{9} \mathrm{Be}[11]$.

In order to show the clustering effects, a simple shell-model is used for calculating nuclear wave functions in comparison with the AMD results. We use a harmonicoscillator model with the potential $M_{N} \omega^{2} r^{2} / 2$. Its wave function is given by $\psi_{n \ell m}=$ $R_{n \ell}(r) Y_{\ell m}(\theta, \phi), \quad R_{n \ell}(r)=N_{n \ell} r^{\ell} e^{-\frac{1}{2} \kappa^{2} r^{2}} L_{n-1}^{\ell+1 / 2}\left(\kappa^{2} r^{2}\right)$, where $Y_{\ell m}(\theta, \phi)$ is the spherical harmonics, $L_{n-1}^{\ell+1 / 2}(x)$ is the Laguerre polynomial, $\kappa$ is defined by $\kappa=\sqrt{M_{N} \omega}$, and $N_{n \ell}$ is the normalization constant. The shell-model density of ${ }^{9} \mathrm{Be}$ is a monotonic distribution, which is totally different from the AMD one in Fig. 4. However, if an angular average is taken for the density, the difference between the AMD and shell model is not very large [12]. Taking the Fourier transformation to the momentum space, we show both densities in Fig. 5 for ${ }^{4} \mathrm{He}$ and ${ }^{9} \mathrm{Be}$. Although the AMD and shell-model densities are almost the same in the ${ }^{4} \mathrm{He}$ nucleus, they are different in ${ }^{9} \mathrm{Be}$. The AMD distribution is shifted toward the high-momentum region, which is caused by the nuclear clustering. Since nucleons are confined mainly in two small space regions (clusters), the high-momentum components are created. It is interesting to find such a difference due to the nuclear clustering. We then estimate its effect on nuclear structure functions $F_{2}^{A}$ by using Eq.(4).

The EMC ratio $\left(F_{2}^{A} / F_{2}^{D}\right)$ is shown for the ${ }^{9}$ Be nucleus in Fig. 6 together with the JLab data. Since effects of short-range correlations and internal nucleon modifications are not included in our estimate, the obtained curves do not fully agree with the data. Here, it is our intention to show the clustering effect in the basic convolution formalism. Considering that the differences between curves are of the order of the experimental errors and there are some theoretical ambiguities, for example, from the correlations

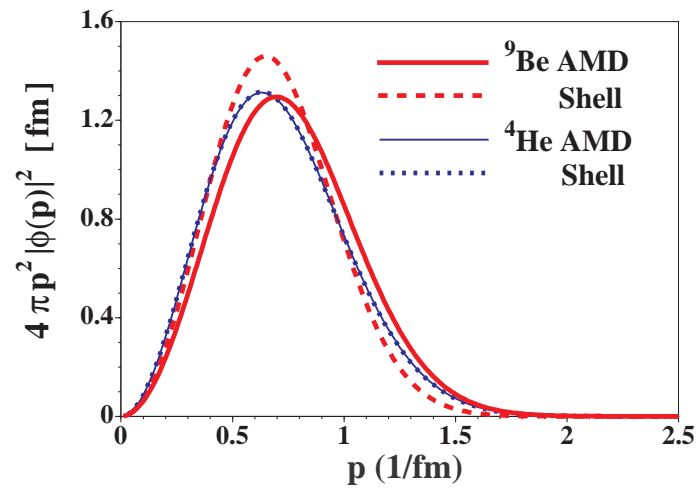

FIGURE 5. Nucleon-momentum distributions in ${ }^{4} \mathrm{He}$ and ${ }^{9} \mathrm{Be}$ by the shell and AMD models [12]. They are obtained by taking Fourier transformations of the coordinate-space densities.

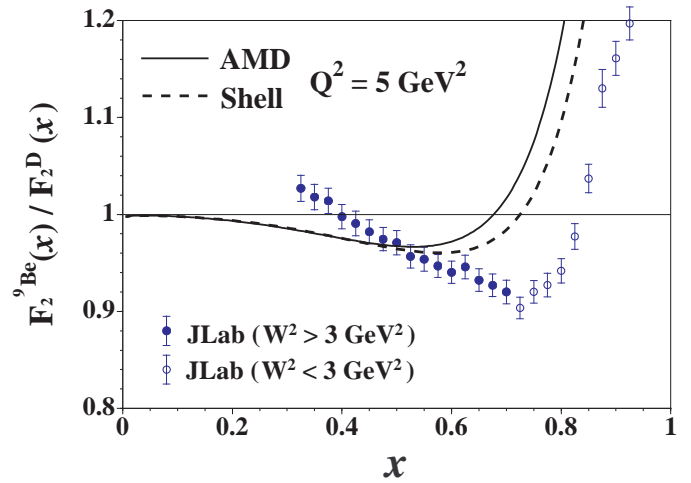

FIGURE 6. Nuclear modifications of $F_{2}$ for ${ }^{9} \mathrm{Be}$ [12]. The dashed and solid curves indicate the shell-model and AMD results at $Q^{2}=5 \mathrm{GeV}^{2}$. JLab measurements are shown for comparison. 


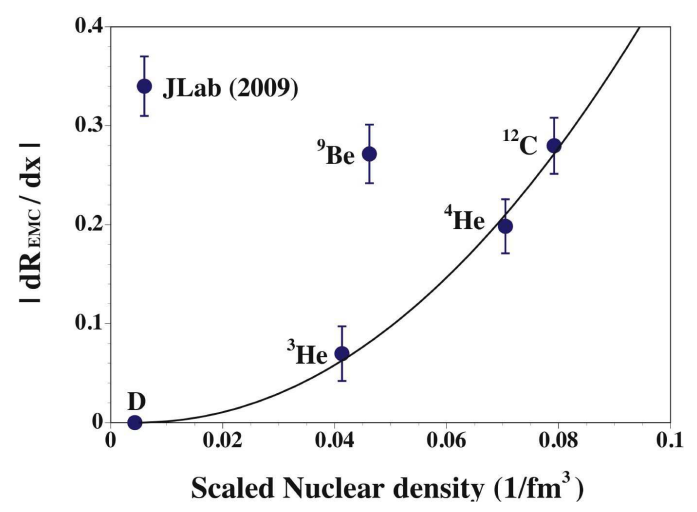

FIGURE 7. Nuclear modification slopes measured at JLab are shown by taking the scaled nuclear density as the abscissa [11]. The curve indicates a fit to the data except for ${ }^{9} \mathrm{Be}$.

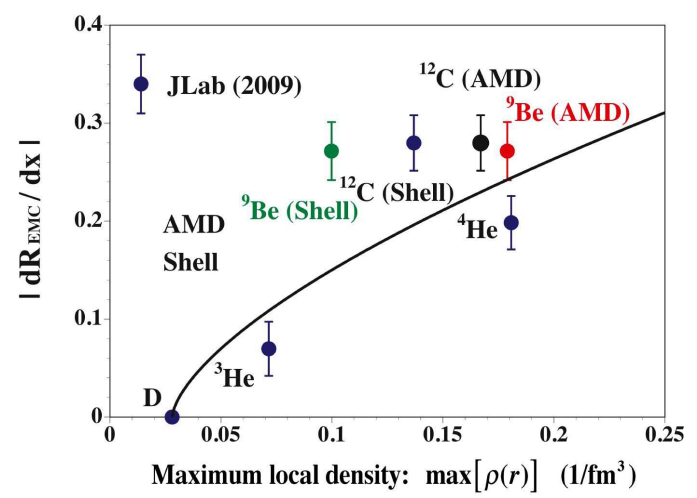

FIGURE 8. Nuclear modification slopes are shown by taking the maximum local densities in the shell and AMD models as the abscissa [12]. The curve indicates a fit to the data points of the shell model except for ${ }^{9} \mathrm{Be}$.

and nucleon modifications if they are included in our estimate, it may not seem easy to find a clear clustering signature.

The Jlab experimental group obtained the nuclear modification slope $d R / d x$ for $R=F_{2}^{A} / F_{2}^{D}$ by approximating their data by straight lines at $0.35<x<0.7$ [11]. They are shown in Fig. 7 as a function of the scaled nuclear density. All the data are along the smooth curve, whereas the ${ }^{9}$ Be slope cannot be explained by such smooth density dependence. However, if the maximum local density is taken as the abscissa, the situation is different as shown in Fig. 8. The curve is plotted to interpolate the shell-model slopes except for ${ }^{9} \mathrm{Be}$. If the maximum density is calculated by the shell model, the modification slope $|d R / d x|$ is too large to be expected from the ${ }^{9} \mathrm{Be}$ density, whereas it is almost on the curve if the maximum density is calculated in the AMD with the clustering structure. The "anomalous" JLab result on the ${ }^{9} \mathrm{Be}$ could be explained by the cluster structure in the nucleus. We also notice a small difference between the AMD and shell model in ${ }^{12} \mathrm{C}$ of Fig. 8, and it is caused by the mixing of a cluster-like configuration in the AMD result of ${ }^{12} \mathrm{C}$.

We would like to clarify our viewpoint because it may be confusing to the reader why the clustering effects appear in $d R / d x$ of Fig. 8, whereas the effects are small in Fig. 6. The nuclear structure functions consist of the mean conventional part and the remaining one depending on the maximum local density. The first part is described by the usual convolution calculation with the spectral function given by the averaged nuclear density distribution, and thus the inhomogeneity of the nuclear density is washed out. The remaining second part is associated with the inhomogeneity of the nuclear density, before taking the average of nuclear wave function, given by the nuclear cluster structure. Our results imply that the physics mechanism, associated with the high densities created by the clusters in ${ }^{9} \mathrm{Be}$, could be the origin for the anomalous nuclear-modification slopes $d R / d x$ of ${ }^{9} \mathrm{Be}$. 


\section{TENSOR STRUCTURE BY QUARK DEGREES OF FREEDOM}

Tensor structure has been investigated in nuclei by hadron degrees of freedom, namely by nucleons and mesons. For example, the $D$-state admixture due to tensor force in nucleon-nucleon interactions is the reason for the finite quadrupole moment in the deuteron. However, the time has come to investigate the tensor structure by quark degrees of freedom. It can be studied by measuring the tensor structure function $b_{1}$ in deep inelastic lepton-deuteron scattering as illustrated in Fig. 9.

\section{Tensor structure $b_{1}$ for deuteron}

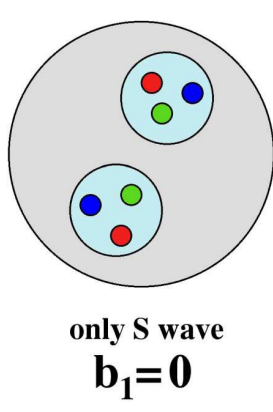

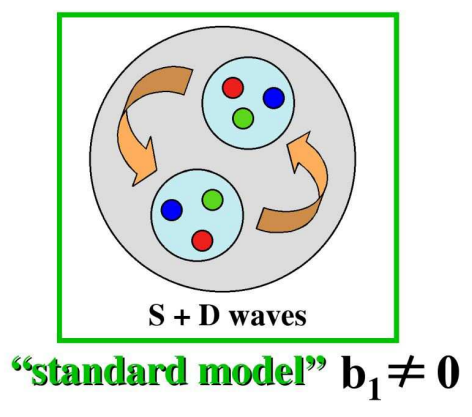

Tensor-structure crisis!?

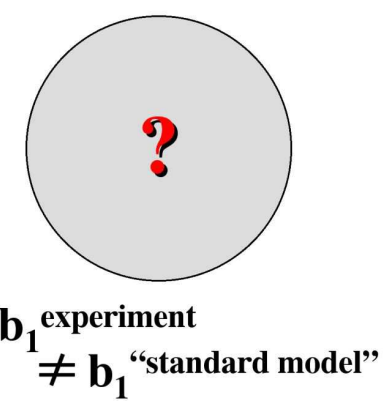

FIGURE 9. Illustration of tensor structure in the deuteron. The structure function $b_{1}$ vanishes if constituents are in the S-wave. A finite $b_{1}$ can be obtained in the conventional deuteron model with the D-state admixture, and it may be considered as the "standard model". Nucleon-spin studies imply that such a conventional picture could fail. Then, a new field of spin physics will be explored by future experimental measurements.

It could be predicted in a conventional nuclear-physics approach with the usual convolution description by PDFs convoluted with nucleon-momentum distributions as illustrated in the middle of Fig. 9. However, our experience of nucleon-spin puzzle suggests that such a traditional model would not work in spin and orbital angular momenta. There is a good possibility that interesting new field of physics will be created by measuring the tensor structure functions.

The structure function $b_{1}$ is expressed by tensor-polarized distributions $\delta_{T} q$ as

$$
b_{1}(x)=\frac{1}{2} \sum_{i} e_{i}^{2}\left[\delta_{T} q_{i}(x)+\delta_{T} \bar{q}_{i}(x)\right], \quad \delta_{T} q_{i}(x)=q_{i}^{0}(x)-\frac{q_{i}^{+1}(x)+q_{i}^{-1}(x)}{2},
$$

where $i$ indicates the flavor of a quark, $e_{i}$ is the charge of the quark, $q_{i}^{\lambda}$ indicates an unpolarized-quark distribution in the hadron spin state $\lambda$, and the distributions are defined by the ones per nucleon. For analyzing HERMES data on $b_{1}$, the distributions are expressed as $\delta_{T} q_{i v}^{D}(x)=\delta_{T} w(x) q_{i v}^{D}(x)$ and $\delta_{T} \bar{q}_{i}^{D}(x)=\alpha_{\bar{q}} \delta_{T} w(x) \bar{q}_{i}^{D}(x)$. Namely, the tensor-polarized distributions are the corresponding unpolarized ones multiplied by a weight function $\delta_{T} w(x)$ and a constant $\alpha_{\bar{q}}$. We consider that a certain fraction of the unpolarized distributions are tensor polarized. In expressing the unpolarized PDFs in the deuteron, nuclear corrections are neglected, isospin symmetry is used for the neutron PDFs in relating them to the proton ones, and flavor-symmetric tensor-polarized 
antiquark distributions are used in $\delta_{T} \bar{q}$. The weight function is then parametrized

$$
\delta_{T} w(x)=a x^{b}(1-x)^{c}\left(x_{0}-x\right),
$$

where a function with a node is taken by considering a constraint based on a quarkparton model: $\int d x b_{1}(x)=-\frac{5}{24} \lim _{t \rightarrow 0} t F_{Q}(t)=0$ [21]. Because of this constraint, $x_{0}$ is expressed by the other parameters which are determined by a $\chi^{2}$ analysis of the HERMES $b_{1}$ data. We tried two different analyses:

- Set 1: Tensor-polarized antiquark distributions are terminated $\left(\alpha_{\bar{q}}=0\right)$.

- Set 2: Finite tensor-polarized antiquark distributions are allowed ( $\alpha_{\bar{q}}$ is a parameter).

The parameters are determined by a $\chi^{2}$ fit to the data in Fig. 10, and obtained $\chi^{2}$ values are $\chi^{2} /$ d.o.f. $=2.83$ and 1.57 for set- 1 and 2, respectively. As shown in Fig. 10, the set1 analysis is not good enough to explain the HERMES data at small $x<0.1$ without the antiquark tensor polarization. The dashed curve is much below the data points, whereas the solid curve can describe the data reasonably well. From these analyses, the determined tensor-polarized distributions for valence quarks and antiquarks are shown in Fig. 11. Of course, the HERMES data are not accurate enough to obtain reliable tensor distributions at this stage; however, the obtained distributions can be used for comparing them with theoretical calculations and for proposed future experiments such as the ones at JLab [22].

The $b_{1}$ sum is expected to vanish in a simple quark-parton model. However, if a deviation is found, it suggests a finite tensor-polarized antiquark distributions [21]:

$$
\begin{aligned}
& \int d x b_{1}(x)=-\frac{5}{24} \lim _{t \rightarrow 0} t F_{Q}(t)+\frac{1}{18} \int d x\left[8 \delta_{T} \bar{u}(x)+2 \delta_{T} \bar{d}(x)+\delta_{T} s(x)+\delta_{T} \bar{s}(x)\right], \\
& \int \frac{d x}{x}\left[F_{2}^{p}(x)-F_{2}^{n}(x)\right]=\frac{1}{3} \int d x\left[u_{v}(x)-d_{v}(x)\right]+\frac{2}{3} \int d x[\bar{u}(x)-\bar{d}(x)] .
\end{aligned}
$$

The $b_{1}$ sum rule is very similar to the Gottfried one [23], whose violation indicated a difference between $\bar{u}$ and $\bar{d}$ by the above equation. An interesting point of our analysis

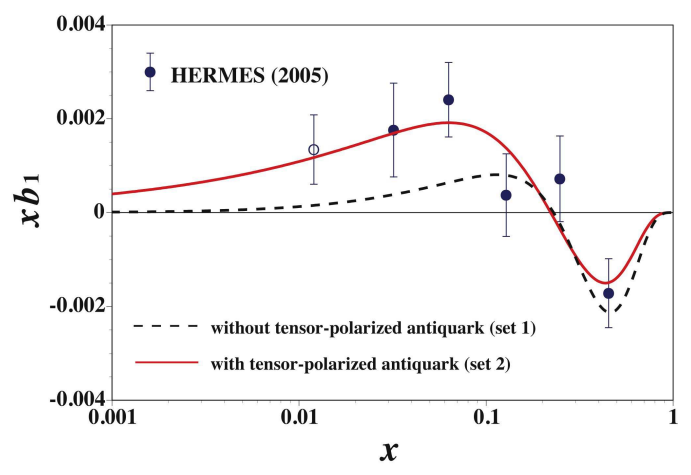

FIGURE 10. Two analysis results are shown with the HERMES $b_{1}$ data [14]. The solid (dashed) curve indicates the parametrization with (without) the antiquark tensor polarization.

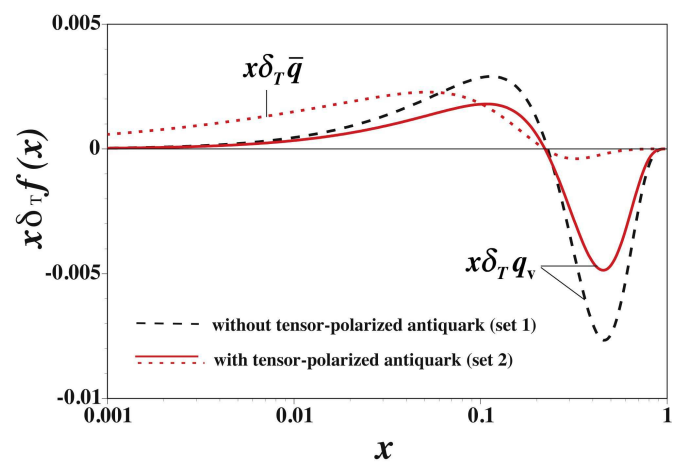

FIGURE 11. Obtained tensor-polarized distributions [14]. The solid and dotted (dashed) curves indicate the distributions with (without) the antiquark tensor polarization. 
[14] is that a finite sum $\int d x b_{1}(x)=0.0058$ was obtained to suggest a finite tensor polarization for antiquarks. It is interesting to conjecture a possible mechanism of this finite antiquark polarization and to test it by an independent experiment. For probing antiquark distributions, Drell-Yan process are ideal. In fact, polarized proton-deuteron Drell-Yan processes are theoretically formulated in Ref. [24] and they could be measured by future hadron facilities such as the J-PARC [25]. The studies of the tensor structure function $b_{1}$ and the tensor-polarized distributions should shed light on a new aspect of high-energy hadron spin physics.

\section{ACKNOWLEDGEMENTS}

The authors thank C. A. Salgado, I. Schienbein, Journal of High Energy Physics, and American Physical Society for permitting them to use figures from their publications.

\section{REFERENCES}

1. D. F. Geesaman, K. Saito, and A. W. Thomas, Ann. Rev. Nucl. Part. Sci. 45, 337 (1995).

2. M. Hirai, S. Kumano, and M. Miyama, Phys. Rev. D 64, 034003 (2001); M. Hirai, S. Kumano, and T.-H. Nagai, Phys. Rev. C 70, 044905 (2004); 76, 065207 (2007).

3. D. de Florian and R. Sassot, Phys. Rev. D 69, 074028 (2004).

4. K. J. Eskola et al., JHEP 0705, 002 (2007); 0807, 102 (2008); 0904, 065 (2009).

5. I. Schienbein et al., Phys. Rev. D 77, 054013 (2008); 80, 094004 (2009); K. Kovarik et al., arXiv:1012.0286.

6. S. A. Kulagin and R. Petti, Nucl. Phys. A 765, 126 (2006); Phys. Rev. D 76, 094023 (2007); Phys. Rev. C 82, 054614 (2010).

7. L. Frankfurt, V. Guzey, and M. Strikman, Phys. Rev. D 71, 054001 (2005); V. Guzey and M. Strikman, Phys. Rev. C 75, 045208 (2007); Phys. Lett. B 687, 167 (2010).

8. G. P. Zeller et al., Phys. Rev. Lett. 88, 091802 (2002); Erratum 90, 239902 (2003).

9. S. Kumano, Phys. Rev. D 66, 111301 (2002); M. Hirai, S. Kumano, and T.-H. Nagai, Phys. Rev. D 71, 113007 (2005); K. J. Eskola and H. Paukkunen, JHEP 0606, 008 (2006).

10. I. C. Cloët, W. Bentz, and A. W. Thomas, Phys. Rev. Lett. 102, 252301 (2009).

11. J. Seely et al., Phys. Rev. Lett. 103, 202301 (2009).

12. M. Hirai, S. Kumano, K. Saito, and T. Watanabe, arXiv:1008.1313 [hep-ph], Phys. Rev. C in press.

13. A. Airapetian et al., Phys. Rev. Lett. 95, 242001 (2005).

14. S. Kumano, Phys. Rev. D 82, 017501 (2010).

15. M. Hirai, S. Kumano, and K. Saito, AIP Conf. Proc. 1189, 269 (2009).

16. D. Dutta, J. C. Peng, I. C. Cloët, and D. Gaskell, arXiv:1007.3916 [nucl-ex].

17. A. D. Martin, R. G. Roberts, W. J. Stirling, and R. S. Thorne, Eur. Phys. J. C 39, 155 (2005).

18. H.-L. Lai et al., JHEP 0704, 089 (2007); A. D. Martin et al., Eur. Phys. J. C 63, 189 (2009); R. D. Ball et al., Nucl. Phys. B 838, 136 (2010).

19. R. B. Wiringa, S. C. Pieper, J. Carlson, and V. R. Pandharipande, Phys. Rev. C 62, 014001 (2000).

20. M. Ericson and S. Kumano, Phys. Rev. C 67, 022201 (2003).

21. F. E. Close and S. Kumano, Phys. Rev. D 42, 2377 (1990).

22. J.-P. Chen, P. Solvignon, N. Kalantarians, O. Rondon, and K. Slifer, Letter of intent to JLab PAC-37.

23. S. Kumano, Phys. Rept. 303, 183 (1998); G. T. Garvey and J.-C. Peng, Prog. Part. Nucl. Phys. 47, $203(2001)$

24. S. Hino and S. Kumano, Phys. Rev. D 59, 094026 (1999); 60, 054018 (1999); S. Kumano and M. Miyama, Phys. Lett. B 479, 149 (2000).

25. See http://j-parc.jp/index-e.html. S. Kumano, Nucl. Phys. A 782, 442 (2007); AIP Conf. Proc. 1056, 444 (2008); arXiv:1010.1596 [hep-ph]. 